\title{
$\Phi$-Admissible Sublinear Singular Operators and Generalized Orlicz-Morrey Spaces
}

\author{
Javanshir J. Hasanov \\ Institute of Mathematics and Mechanics, Baku, Azerbaijan \\ Correspondence should be addressed to Javanshir J. Hasanov; hasanovjavanshir@yahoo.com.tr \\ Received 13 August 2013; Revised 19 October 2013; Accepted 21 October 2013; Published 22 January 2014 \\ Academic Editor: Yoshihiro Sawano
}

Copyright (c) 2014 Javanshir J. Hasanov. This is an open access article distributed under the Creative Commons Attribution License, which permits unrestricted use, distribution, and reproduction in any medium, provided the original work is properly cited.

We study the boundedness of $\Phi$-admissible sublinear singular operators on Orlicz-Morrey spaces $M_{\Phi, \varphi}\left(\mathbb{R}^{n}\right)$. These conditions are satisfied by most of the operators in harmonic analysis, such as the Hardy-Littlewood maximal operator and Calderón-Zygmund singular integral operator.

\section{Introduction}

As it is well known that Morrey [1] introduced the classical Morrey spaces to investigate the local behavior of solutions to second-order elliptic partial differential equations (PDE), we recall its definition as

$$
\begin{aligned}
M_{p, \lambda}\left(\mathbb{R}^{n}\right)= & \left\{f \in L_{p}^{\text {loc }}\left(\mathbb{R}^{n}\right):\right. \\
& \left.\|f\|_{M_{p, \lambda}}:=\sup _{x \in \mathbb{R}^{n}, r>0} r^{-\lambda / p}\|f\|_{L_{p}(B(x, r))}<\infty\right\},
\end{aligned}
$$

where $0 \leq \lambda \leq n, 1 \leq p<\infty$. Here and everywhere in the sequel $B(x, r)$ stands for the ball in $\mathbb{R}^{n}$ of radius $r$ centered at $x$. Let $|B(x, r)|$ be the Lebesgue measure of the ball $B(x, r)$ and $|B(x, r)|=v_{n} r^{n}$, where $v_{n}=|B(0,1)| . M_{p, \lambda}\left(\mathbb{R}^{n}\right)$ was an expansion of $L_{p}\left(\mathbb{R}^{n}\right)$ in the sense that $M_{p, 0}\left(\mathbb{R}^{n}\right)=L_{p}\left(\mathbb{R}^{n}\right)$. We also denote by $W M_{p, \lambda} \equiv W M_{p, \lambda}\left(\mathbb{R}^{n}\right)$ the weak Morrey space of all functions $f \in W L_{\Phi}^{\text {loc }}\left(\mathbb{R}^{n}\right)$ for which

$$
\|f\|_{W M_{p, \lambda}}=\sup _{x \in \mathbb{R}^{n}, r>0} r^{-\lambda / p}\|f\|_{W L_{p}(B(x, r))}<\infty,
$$

where $W L_{p}(B(x, r))$ denotes the weak $L_{p}$-space (for $L_{\Phi}^{\text {loc }}\left(\mathbb{R}^{n}\right)$ see Definition 4). Morrey found that many properties of solutions to $\mathrm{PDE}$ can be attributed to the boundedness of some operators on Morrey spaces. Maximal functions and singular integrals play a key role in harmonic analysis since maximal functions could control crucial quantitative information concerning the given functions, despite their larger size, while singular integrals, Hilbert transform as its prototype, nowadays intimately connected with PDE, operator theory and other fields.

Let $f \in L_{1}^{\text {loc }}\left(\mathbb{R}^{n}\right)$. The Hardy-Littlewood (H-L) maximal function of $f$ is defined by

$$
M f(x)=\sup _{r>0} \frac{1}{|B(x, r)|} \int_{B(x, r)}|f(y)| d y .
$$

The Calderón-Zygmund (C-Z) singular integral operator is defined by

$$
T f(x)=\int_{\mathbb{R}^{n}} K(x, y) f(y) d y
$$


and bounded on $L_{2}\left(\mathbb{R}^{n}\right)$, where $K(x, y)$ is a "standard singular kernel," that is, a continuous function defined on $\left\{(x, y) \in \mathbb{R}^{n} \times \mathbb{R}^{n}: x \neq y\right\}$ and satisfying the estimates

$$
\begin{aligned}
|K(x, y)| \leq C|x-y|^{-n} \quad \forall x \neq y, \\
|K(x, y)-K(x, z)| \leq C \frac{|y-z|^{\sigma}}{|x-y|^{n+\sigma}}, \quad \sigma>0, \\
\quad \text { if }|x-y|>2|y-z|, \\
|K(x, y)-K(\xi, y)| \leq C \frac{|x-\xi|^{\sigma}}{|x-y|^{n+\sigma}}, \quad \sigma>0, \\
\text { if }|x-y|>2|x-\xi| .
\end{aligned}
$$

It is well known that the maximal and singular integral operators play an important role in harmonic analysis (see $[2,3])$.

Orlicz spaces, introduced in $[4,5]$, are generalizations of Lebesgue spaces $L_{p}$ (see also, [6-8]). They are useful tools in harmonic analysis and its applications. For example, the Hardy-Littlewood maximal operator is bounded on $L_{p}$ for $1<p<\infty$, but not on $L_{1}$. Using Orlicz spaces, we can investigate the boundedness of the maximal operator near $p=1$ more precisely (see [9-11]).

We find it convenient to define the generalized OrliczMorrey spaces in the following form (see Definition 3 for the notion of Young functions).

Definition 1. Let $\varphi(x, r)$ be a positive measurable function on $\mathbb{R}^{n} \times(0, \infty)$ and $\Phi$ a Young function. We define the generalized Orlicz-Morrey space $M_{\Phi, \varphi}\left(\mathbb{R}^{n}\right)$ as the space of all functions $f \in L_{\Phi}^{\text {loc }}\left(\mathbb{R}^{n}\right)$ with finite quasinorm

$$
\|f\|_{M_{\Phi, \varphi}}=\sup _{x \in \mathbb{R}^{n}, r>0} \varphi(x, r)^{-1}\|f\|_{L_{\Phi}(B(x, r))^{.}}
$$

Remark 2. The Calderón-Zygmund (C-Z) singular integral operators $T$ are $L_{2}$ bounded and expressed as (4) for all $f \in$ $C_{\text {comp }}^{\infty}\left(\mathbb{R}^{n}\right)$, with standard kernel $K$. Then, one can prove that $T$ is of weak type $(1,1)$ and type $(p, p), 1<p<\infty$, for $f \in C_{\text {comp }}^{\infty}\left(\mathbb{R}^{n}\right)$, and then $T$ is uniquely extended to an $L_{p^{-}}$ bounded operator by the density of $C_{\text {comp }}^{\infty}\left(\mathbb{R}^{n}\right)$ in $L_{p}\left(\mathbb{R}^{n}\right)$. On the other hand, $C_{\text {comp }}^{\infty}\left(\mathbb{R}^{n}\right)$ is not dense in Morrey spaces in general. Therefore, we need to give a precise definition of $T f$ for the function $f$ in Morrey spaces; for example,

$$
T f(x)=T\left(f \chi_{2 B}\right)+\int_{\mathbb{R}^{n} \backslash(2 B)} K(x, y) f(y) d y,
$$

for some ball $B$ which contains $x$, with proving the absolutely convergence of the integral in the second term and the independence of the choice of the ball $B$ (see $[12,13]$ for example). Also, $C_{\text {comp }}^{\infty}\left(\mathbb{R}^{n}\right)$ is dense in Orlicz spaces $L_{\Phi}\left(\mathbb{R}^{n}\right)$ if and only if $\Phi$ satisfies the $\Delta_{2}$ condition.

The main purpose of this paper is to find sufficient conditions on general Young function $\Phi$ and the functions $\varphi_{1}, \varphi_{2}$ ensuring that the sublinear operators generated by singular integral operators are of weak or strong type from generalized Orlicz-Morrey spaces $M_{\Phi, \varphi_{1}}\left(\mathbb{R}^{n}\right)$ into $M_{\Phi, \varphi_{2}}\left(\mathbb{R}^{n}\right)$. Note that the Orlicz-Morrey spaces were introduced and studied by Nakai in $[12,14]$. Also the boundedness of the operators of harmonic analysis on Orlicz-Morrey spaces see also, $[9,10,13-17]$.

By $A \lesssim B$, we mean that $A \leq C B$ with some positive constant $C$ independent of appropriate quantities. If $A \leqslant B$ and $B \lesssim A$, we write $A \approx B$ and say that $A$ and $B$ are equivalent.

\section{Preliminaries}

Definition 3. A function $\Phi:[0,+\infty] \rightarrow[0,+\infty]$ is called a Young function if $\Phi$ is convex, left-continuous, $\lim _{r \rightarrow+0} \Phi(r)=\Phi(0)=0$ and $\lim _{r \rightarrow+\infty} \Phi(r)=\Phi(+\infty)=$ $+\infty$.

From the convexity and $\Phi(0)=0$, it follows that any Young function is increasing. If there exists $s \in(0,+\infty)$ such that $\Phi(s)=+\infty$, then $\Phi(r)=+\infty$ for $r \geq s$.

We say that $\Phi \in \Delta_{2}$ if, for any $a>1$, there exists a constant $C_{a}>0$ such that $\Phi(a t) \leq C_{a} \Phi(t)$ for all $t>0$.

Recall that a function $\Phi$ is said to be quasiconvex if there exist a convex function $\omega$ and a constant $c>0$ such that

$$
\omega(t) \leq \Phi(t) \leq c \omega(c t), \quad t \in[0,+\infty) .
$$

Let $y$ be the set of all Young functions $\Phi$ such that

$$
0<\Phi(r)<+\infty \quad \text { for } 0<r<+\infty
$$

If $\Phi \in \mathcal{Y}$, then $\Phi$ is absolutely continuous on every closed interval in $[0,+\infty)$ and bijective from $[0,+\infty)$ to itself.

Definition 4 (Orlicz space). For a Young function $\Phi$, the set

$$
\begin{gathered}
L_{\Phi}\left(\mathbb{R}^{n}\right)=\left\{f \in L_{1}^{\text {loc }}\left(\mathbb{R}^{n}\right): \int_{\mathbb{R}^{n}} \Phi(k|f(x)|) d x<+\infty\right. \\
\text { for some } k>0\}
\end{gathered}
$$

is called Orlicz space. The space $L_{\Phi}^{\text {loc }}\left(\mathbb{R}^{n}\right)$ endowed with the natural topology is defined as the set of all functions $f$ such that $f \chi_{B} \in L_{\Phi}\left(\mathbb{R}^{n}\right)$ for all balls $B \subset \mathbb{R}^{n}$.

Note that $L_{\Phi}\left(\mathbb{R}^{n}\right)$ is a Banach space with respect to the norm

$$
\|f\|_{L_{\Phi}}=\inf \left\{\lambda>0: \int_{\mathbb{R}^{n}} \Phi\left(\frac{|f(x)|}{\lambda}\right) d x \leq 1\right\} ;
$$

see, for example, [18, Section 3, Theorem 10], so that

$$
\int_{\mathbb{R}^{n}} \Phi\left(\frac{|f(x)|}{\|f\|_{L_{\Phi}}}\right) d x \leq 1
$$


Definition 5. The weak Orlicz space

$$
W L_{\Phi}\left(\mathbb{R}^{n}\right)=\left\{f \in L_{1 \mathrm{oc}}^{1}\left(\mathbb{R}^{n}\right):\|f\|_{W L_{\Phi}}<+\infty\right\}
$$

is defined by the norm

$$
\|f\|_{W L_{\Phi}}=\inf \left\{\lambda>0: \sup _{t>0} \Phi(t) m\left(\frac{f}{\lambda}, t\right) \leq 1\right\},
$$

where $m(f, t)=\left|\left\{x \in \mathbb{R}^{n}:|f(x)|>t\right\}\right|$.

For Young functions $\Phi$ and $\Psi$, we write $\Phi \sim \Psi$ if there exists a constant $C \geq 1$ such that

$$
\Phi\left(C^{-1} r\right) \leq \Psi(r) \leq \Phi(C r) \quad \forall r \geq 0 .
$$

If $\Phi \approx \Psi$, then $L_{\Phi}\left(\mathbb{R}^{n}\right)=L_{\Psi}\left(\mathbb{R}^{n}\right)$ with equivalent norms.

For a Young function $\Phi$ and $0 \leq s \leq+\infty$, let

$$
\Phi^{-1}(s)=\inf \{r \geq 0: \Phi(r)>s\} \quad(\inf \emptyset=+\infty) .
$$

If $\Phi \in \mathcal{Y}$, then $\Phi^{-1}$ is the usual inverse function of $\Phi$. We note that

$$
\Phi\left(\Phi^{-1}(r)\right) \leq r \leq \Phi^{-1}(\Phi(r)) \text { for } 0 \leq r<+\infty .
$$

A Young function $\Phi$ is said to satisfy the $\nabla_{2}$-condition, denoted also by $\Phi \in \nabla_{2}$, if

$$
\Phi(r) \leq \frac{1}{2 k} \Phi(k r), \quad r \geq 0,
$$

for some $k>1$.

For a Young function $\Phi$, the complementary function $\widetilde{\Phi}(r)$ is defined by

$$
\widetilde{\Phi}(r)= \begin{cases}\sup \{r s-\Phi(s): s \in[0, \infty)\}, & r \in[0, \infty) \\ +\infty, & r=+\infty .\end{cases}
$$

The complementary function $\widetilde{\Phi}$ is also a Young function and $\widetilde{\widetilde{\Phi}}=\Phi$. If $\Phi(r)=r$, then $\widetilde{\Phi}(r)=0$ for $0 \leq r \leq 1$ and $\widetilde{\Phi}(r)=$ $+\infty$ for $r>1$. If $1<p<\infty, 1 / p+1 / p^{\prime}=1$, and $\Phi(r)=$ $r^{p} / p$, then $\widetilde{\Phi}(r)=r^{p^{\prime}} / p^{\prime}$. If $\Phi(r)=e^{r}-r-1$, then $\widetilde{\Phi}(r)=$ $(1+r) \log (1+r)-r$.

It is known that

$$
r \leq \Phi^{-1}(r) \widetilde{\Phi}^{-1}(r) \leq 2 r \text { for } r \geq 0 .
$$
$|T g|$.

Let $T$ be a sublinear operator; that is, $|T(f+g)| \leq|T f|+$

Definition 6 ( $\Phi$-admissible sublinear singular operator). Let $\Phi$ be any Young function. A sublinear operator $T$ will be called $\Phi$-admissible singular operator, if

(1) $T$ satisfies the size condition of the form

$$
\begin{aligned}
& \chi_{B(x, r)}(z)\left|T\left(f \chi_{\mathbb{R}^{n} \backslash B(x, 2 r)}\right)(z)\right| \\
& \quad \leq C \chi_{B(x, r)} \int_{\mathbb{R}^{n} \backslash B(x, 2 r)} \frac{|f(y)|}{|y-z|^{n}} d y
\end{aligned}
$$

for $x \in \mathbb{R}^{n}$ and $r>0$;

(2) $T$ is bounded in $L \Phi(\mathbb{R} n)$.
In the case $\Phi(r)=r^{p}$ the $\Phi$-admissible singular operator will be called the $p$-admissible singular operator.

Definition 7 (weak $\Phi$-admissible sublinear singular operator). Let $\Phi$ be any Young function. A sublinear operator $T$ will be called the weak $\Phi$-admissible singular operator, if

(1) $T$ satisfies the size condition (21);

(2) $T$ is bounded from $L_{\Phi}\left(\mathbb{R}^{n}\right)$ to the weak $W L_{\Phi}\left(\mathbb{R}^{n}\right)$.

In the case $\Phi(r)=r^{p}$ the weak $\Phi$-admissible singular operator will be called weak $p$-admissible singular operator.

Necessary and sufficient conditions on $\Phi$ for the boundedness of $M$ in Orlicz spaces $L_{\Phi}\left(\mathbb{R}^{n}\right)$ have been obtained in [19, Theorem 2.1] and [20, Theorem 1.2.1]. Note that $\Phi \in \nabla_{2}$ if and only if $\widetilde{\Phi} \in \Delta_{2}$. Also, if $\Phi$ is a Young function, then $\Phi \in \nabla_{2}$ if and only if $\Phi^{\gamma}$ is quasiconvex for some $\gamma \in(0,1)$ (see, e.g., [20, page 15]). With this remark taken into account, the known boundedness statement runs as follows.

The following theorem was in fact proved in [11].

Theorem 8 (see [11]). Let $\Phi$ be any Young function. Then the maximal operator $M$ is bounded from $L_{\Phi}\left(\mathbb{R}^{n}\right)$ to $W L_{\Phi}\left(\mathbb{R}^{n}\right)$ and for $\Phi \in \nabla_{2}$ bounded in $L_{\Phi}\left(\mathbb{R}^{n}\right)$.

Sufficient conditions on $\Phi$ for the boundedness of the singular integral operator $T$ in Orlicz spaces $L_{\Phi}\left(\mathbb{R}^{n}\right)$ are known; see [20, Theorem 1.4.3] and [17, Theorem 3.3]. The following theorem was proved in [21].

Theorem 9 (see [21]). Let $\Phi$ be a Young function and let $T$ be a singular integral operator. If $\Phi \in \Delta_{2} \bigcap \nabla_{2}$, then the operator $T$ is bounded on $L_{\Phi}\left(\mathbb{R}^{n}\right)$ and if $\Phi \in \Delta_{2}$, then the operator $T$ is bounded from $L_{\Phi}\left(\mathbb{R}^{n}\right)$ to $W L_{\Phi}\left(\mathbb{R}^{n}\right)$.

Remark 10. Note that, from Theorems 8 and 9 we get the any Young function $\Phi$ the maximal operator $M$ and for the Young function $\Phi \in \Delta_{2}$ the singular integral operator $T$ are the weak $\Phi$-admissible singular operator. Also for the Young function $\Phi \in \nabla_{2}$ the maximal operator $M$ and for the Young function $\Phi \in \Delta_{2} \cap \nabla_{2}$ the singular integral operator $T$ are the $\Phi$ admissible singular operator.

Definition 11 (generalized Orlicz-Morrey space). Let $\varphi(x, r)$ be a positive measurable function on $\mathbb{R}^{n} \times(0, \infty)$ and $\Phi$ be any Young function. We denote by $M_{\Phi, \varphi}\left(\mathbb{R}^{n}\right)$ the generalized Orlicz-Morrey space, the space of all functions $f \in L_{\Phi}^{\text {loc }}\left(\mathbb{R}^{n}\right)$ with finite quasinorm

$$
\|f\|_{M_{\Phi, \varphi}}=\sup _{x \in \mathbb{R}^{n}, r>0} \varphi(x, r)^{-1}\|f\|_{L_{\Phi}(B(x, r))} .
$$

Also, by $W M_{\Phi, \varphi}\left(\mathbb{R}^{n}\right)$ we denote the weak generalized OrliczMorrey space of all functions $f \in W L_{\Phi}^{\text {loc }}\left(\mathbb{R}^{n}\right)$ for which

$$
\|f\|_{W M_{\Phi, \varphi}}=\sup _{x \in \mathbb{R}^{n}, r>0} \varphi(x, r)^{-1}\|f\|_{W L_{\Phi}(B(x, r))}<\infty .
$$


According to this definition, we recover the Orlicz space $L_{\Phi}$ and weak Orlicz space $W L_{\Phi}$ under the choice $\varphi \equiv 1$ :

$$
\begin{gathered}
L_{\Phi}=\left.M_{\Phi, \varphi}\right|_{\varphi \equiv 1}, \\
W L_{\Phi}=\left.W M_{\Phi, \varphi}\right|_{\varphi \equiv 1} .
\end{gathered}
$$

Also according to this definition, we recover the generalized Morrey space $M_{p, \varphi}$ and weak generalized Morrey space $W M_{p, \varphi}$ under the choice $\Phi(r)=r^{p}$ :

$$
\begin{gathered}
M_{p, \varphi}=\left.M_{\Phi, \varphi}\right|_{\Phi(r)=r^{p}}, \\
W M_{p, \varphi}=\left.W M_{\Phi, \varphi}\right|_{\Phi(r)=r^{p}} .
\end{gathered}
$$

The following statement, containing Guliyev results obtained in [22-24], was proved in [25] (see also [26]).

Theorem 12. Let $1 \leq p<\infty$, and $\left(\varphi_{1}, \varphi_{2}\right)$ satisfies the condition

$$
\int_{r}^{\infty} \frac{\text { ess inf }_{t<s<\infty} \varphi_{1}(x, s) s^{n / p}}{t^{(n / p)+1}} d t \leq C \varphi_{2}(x, r),
$$

where $C$ does not depend on $x$ and $r$. Then, for $1<p<$ $\infty$ a p-admissible sublinear singular operator $T$ is bounded from $M_{p, \varphi_{1}}\left(\mathbb{R}^{n}\right)$ to $M_{p, \varphi_{2}}\left(\mathbb{R}^{n}\right)$ and for $1 \leq p<\infty$ a weak p-admissible sublinear singular operator $T$ is bounded from $M_{p, \varphi_{1}}\left(\mathbb{R}^{n}\right)$ to $W M_{p, \varphi_{2}}\left(\mathbb{R}^{n}\right)$.

We will use the following statement on the boundedness of the weighted Hardy operator:

$$
H_{w}^{*} g(t):=\int_{t}^{\infty} g(s) w(s) d s, \quad 0<t<\infty,
$$

where $w$ is a weight.

The following theorem was proved in [27] (see also, [28]).

Theorem 13. Let $v_{1}, v_{2}$ and $w$ be weights on $(0, \infty)$ and $v_{1}(t)$ be bounded outside a neighborhood of the origin. The inequality

$$
\sup _{t>0} v_{2}(t) H_{w}^{*} g(t) \leq C \sup _{t>0} v_{1}(t) g(t)
$$

holds for some $C>0$ for all non-negative and non-decreasing $g$ on $(0, \infty)$ if and only if

$$
B:=\sup _{t>0} v_{2}(t) \int_{t}^{\infty} \frac{w(s) d s}{\sup _{s<\tau<\infty} v_{1}(\tau)}<\infty .
$$

Moreover, the value $C=B$ is the best constant for (28).

Remark 14. In (28) and (29) it is assumed that $1 / \infty=0$ and $0 \cdot \infty=0$.

\section{3. $\Phi$-Admissible Sublinear Singular Operator in the Spaces $M_{\Phi, \varphi}$}

In this section, sufficient conditions on $\varphi$ for the boundedness of the $\Phi$-admissible sublinear singular operator $T$ in generalized Orlicz-Morrey spaces $M_{\Phi, \varphi}\left(\mathbb{R}^{n}\right)$ are obtained.

The following lemma was generalizations of the Guliyev lemma for Orlicz spaces [22-24].
Lemma 15. Let $\Phi$ be any Young function and $f \in L_{\Phi}^{\text {loc }}\left(\mathbb{R}^{n}\right)$, $B=B\left(x_{0}, r\right), x_{0} \in \mathbb{R}^{n}$ and $r>0$. Then for the $\Phi$-admissible sublinear singular operator $T$ the following inequality is valid

$$
\begin{aligned}
\|T f\|_{L_{\Phi}(B)} \leqslant & \frac{1}{\Phi^{-1}\left(r^{-n}\right)} \\
& \times \int_{2 r}^{\infty}\|f\|_{L_{\Phi}\left(B\left(x_{0}, t\right)\right)} \Phi^{-1}\left(t^{-n}\right) \frac{d t}{t},
\end{aligned}
$$

and for the weak $\Phi$-admissible sublinear singular operator $T$ the following inequality is valid

$$
\|T f\|_{W L_{\Phi}(B)} \lesssim \frac{1}{\Phi^{-1}\left(r^{-n}\right)} \int_{2 r}^{\infty}\|f\|_{L_{\Phi}\left(B\left(x_{0}, t\right)\right)} \Phi^{-1}\left(t^{-n}\right) \frac{d t}{t} .
$$

Here $\Phi^{-1}(r)$ defined in (16).

Proof. Let $\Phi$ be any Young function and the operator $T$ be a $\Phi$-admissible sublinear singular operator. With the notation $2 B=B\left(x_{0}, 2 r\right)$, we represent $f$ as

$$
\begin{aligned}
& f=f_{1}+f_{2}, \quad f_{1}(y)=f(y) \chi_{2 B}(y), \\
& f_{2}(y)=f(y) \chi^{\complement}(2 B)(y),
\end{aligned}
$$

and then

$$
\|T f\|_{L_{\Phi}(B)} \leq\left\|T f_{1}\right\|_{L_{\Phi}(B)}+\left\|T f_{2}\right\|_{L_{\Phi}(B)} .
$$

Since $f_{1} \in L_{\Phi}\left(\mathbb{R}^{n}\right)$, by the boundedness of $T$ in $L_{\Phi}\left(\mathbb{R}^{n}\right)$, it follows that

$$
\left\|T f_{1}\right\|_{L_{\Phi}(B)} \leq\left\|T f_{1}\right\|_{L_{\Phi}\left(\mathbb{R}^{n}\right)} \leq C\left\|f_{1}\right\|_{L_{\Phi}\left(\mathbb{R}^{n}\right)}=C\|f\|_{L_{\Phi}(2 B)} .
$$

Next, observe that $x \in B, y \in{ }^{\complement}(2 B)$ imply $(1 / 2)\left|x_{0}-y\right| \leq$ $|x-y| \leq(3 / 2)\left|x_{0}-y\right|$. We get

$$
\left|T f_{2}(x)\right| \leq C \int_{C_{(2 B)}} \frac{|f(y)|}{\left|x_{0}-y\right|^{n}} d y .
$$

By Fubini's theorem, we have

$$
\begin{aligned}
\int_{(2 B)} \frac{|f(y)|}{\left|x_{0}-y\right|^{n}} d y & \approx \int_{\mathcal{C}_{(2 B)}}|f(y)| \int_{\left|x_{0}-y\right|}^{\infty} \frac{d t}{t^{n+1}} d y \\
& \approx \int_{2 r}^{\infty} \int_{2 r \leq\left|x_{0}-y\right|<t}|f(y)| d y \frac{d t}{t^{n+1}} \\
& \lesssim \int_{2 r}^{\infty} \int_{B\left(x_{0}, t\right)}|f(y)| d y \frac{d t}{t^{n+1}} .
\end{aligned}
$$

Applying the following Hölder's inequality

$$
\|f\|_{L_{1}(B)} \leq 2\|1\|_{L_{\widetilde{\Phi}}(B)}\|f\|_{L_{\Phi}(B)}
$$


we get

$$
\begin{aligned}
& \int_{\mathcal{C}_{(2 B)}} \frac{|f(y)|}{\left|x_{0}-y\right|^{n}} d y \\
& \quad \lesssim \int_{2 r}^{\infty}\|f\|_{L_{\Phi}\left(B\left(x_{0}, t\right)\right)}\|1\|_{L_{\widetilde{\Phi}}\left(B\left(x_{0}, t\right)\right)} \frac{d t}{t^{n+1}} \\
& \quad=\int_{2 r}^{\infty}\|f\|_{L_{\Phi}\left(B\left(x_{0}, t\right)\right)} \frac{1}{\widetilde{\Phi}^{-1}\left(\left|B\left(x_{0}, t\right)\right|^{-1}\right)} \frac{d t}{t^{n+1}} \\
& \approx \int_{2 r}^{\infty}\|f\|_{L_{\Phi}\left(B\left(x_{0}, t\right)\right)} \Phi^{-1}\left(t^{-n}\right) \frac{d t}{t} .
\end{aligned}
$$

Moreover,

$$
\begin{aligned}
\left\|T f_{2}\right\|_{W L_{\Phi}(B)} & \leq\left\|T f_{2}\right\|_{L_{\Phi}(B)} \\
& \leq \frac{1}{\Phi^{-1}\left(r^{-n}\right)} \int_{2 r}^{\infty}\|f\|_{L_{\Phi}\left(B\left(x_{0}, t\right)\right)} \Phi^{-1}\left(t^{-n}\right) \frac{d t}{t}
\end{aligned}
$$

Thus,

$$
\begin{aligned}
\|T f\|_{L_{\Phi}(B)} \lesssim & \|f\|_{L_{\Phi}(2 B)}+\frac{1}{\Phi^{-1}\left(r^{-n}\right)} \\
& \times \int_{2 r}^{\infty}\|f\|_{L_{\Phi}\left(B\left(x_{0}, t\right)\right)} \Phi^{-1}\left(t^{-n}\right) \frac{d t}{t} .
\end{aligned}
$$

On the other hand, by (20) we get

$$
\begin{aligned}
\|f\|_{L_{\Phi}(2 B)} & \approx \frac{1}{\Phi^{-1}\left(r^{-n}\right)}\|f\|_{L_{\Phi}(2 B)} \Phi^{-1}\left(r^{-n}\right) r^{n} \int_{2 r}^{\infty} \frac{d t}{t^{n+1}} \\
& \leq \frac{1}{\Phi^{-1}\left(r^{-n}\right)}\|f\|_{L_{\Phi}(2 B)} r^{n} \int_{2 r}^{\infty} \Phi^{-1}\left(t^{-n}\right) \frac{d t}{t^{n+1}} \\
& \leq \frac{1}{\Phi^{-1}\left(r^{-n}\right)}\|f\|_{L_{\Phi}(2 B)} \int_{2 r}^{\infty} \Phi^{-1}\left(t^{-n}\right) \frac{d t}{t} \\
& \leq \int_{2 r}^{\infty}\|f\|_{L_{\Phi}\left(B\left(x_{0}, t\right)\right)} \Phi^{-1}\left(t^{-n}\right) \frac{d t}{t}
\end{aligned}
$$

and then

$$
\|f\|_{L_{\Phi}(2 B)} \lesssim \frac{1}{\Phi^{-1}\left(r^{-n}\right)} \int_{2 r}^{\infty}\|f\|_{L_{\Phi}\left(B\left(x_{0}, t\right)\right)} \Phi^{-1}\left(t^{-n}\right) \frac{d t}{t} .
$$

Thus,

$$
\|T f\|_{L_{\Phi}(B)} \lesssim \frac{1}{\Phi^{-1}\left(r^{-n}\right)} \int_{2 r}^{\infty}\|f\|_{L_{\Phi}\left(B\left(x_{0}, t\right)\right)} \Phi^{-1}\left(t^{-n}\right) \frac{d t}{t} .
$$

Let the operator $T$ be a weak $\Phi$-admissible sublinear singular operator. Then, by the weak boundedness of $T$ on Orlicz space and (39) it follows that

$$
\begin{aligned}
\left\|T f_{1}\right\|_{W L_{\Phi}(B)} & \leq\left\|T f_{1}\right\|_{W L_{\Phi}\left(\mathbb{R}^{n}\right)} \lesssim\left\|f_{1}\right\|_{L_{\Phi}\left(\mathbb{R}^{n}\right)}=\|f\|_{L_{\Phi}(2 B)} \\
& \leqslant \frac{1}{\Phi^{-1}\left(r^{-n}\right)} \int_{2 r}^{\infty} \int_{B\left(x_{0}, t\right)}|f(y)| d y \frac{d t}{t^{n+1}} .
\end{aligned}
$$

Then by (39) and (44), we get inequality (31).

Corollary 16 (see [15]). Let $\Phi$ be any Young function and $f \in$ $L_{\Phi}^{\text {loc }}\left(\mathbb{R}^{n}\right), B=B\left(x_{0}, r\right), x_{0} \in \mathbb{R}^{n}$, and $r>0$. Then, for the singular integral operator $T$ the following inequalities are valid:

$$
\|T f\|_{L_{\Phi}(B)} \lesssim \frac{1}{\Phi^{-1}\left(r^{-n}\right)} \int_{2 r}^{\infty}\|f\|_{L_{\Phi}\left(B\left(x_{0}, t\right)\right)} \Phi^{-1}\left(t^{-n}\right) \frac{d t}{t},
$$

if $\Phi \in \Delta_{2} \cap \nabla_{2}$ and

$$
\|T f\|_{W L_{\Phi}(B)} \lesssim \frac{1}{\Phi^{-1}\left(r^{-n}\right)} \int_{2 r}^{\infty}\|f\|_{L_{\Phi}\left(B\left(x_{0}, t\right)\right)} \Phi^{-1}\left(t^{-n}\right) \frac{d t}{t},
$$

if $\Phi \in \Delta_{2}$.

Corollary 17 (see [22-24]). Let $1 \leq p<\infty$ and $f \in L_{p}^{\text {loc }}\left(\mathbb{R}^{n}\right)$, $B=B\left(x_{0}, r\right), x_{0} \in \mathbb{R}^{n}$, and $r>0$. Then, for the $p$-admissible sublinear singular operator $T$ the following inequality is valid:

$$
\|T f\|_{L_{p}\left(B\left(x_{0}, r\right)\right)} \leqslant r^{n / p} \int_{2 r}^{\infty} t^{-(n / p)-1}\|f\|_{L_{p}\left(B\left(x_{0}, t\right)\right)} d t,
$$

$$
1<p<\infty
$$

and for the weak $p$-admissible sublinear singular operator $T$ the following inequality is valid

$$
\|T f\|_{W L_{p}\left(B\left(x_{0}, r\right)\right)} \lesssim r^{n} \int_{2 r}^{\infty} t^{-n-1}\|f\|_{L_{p}\left(B\left(x_{0}, t\right)\right)} d t, \quad 1 \leq p<\infty .
$$

Theorem 18. Let $\Phi$ be any Young function and $\varphi_{1}, \varphi_{2}$, and $\Phi$ satisfy the condition

$$
\begin{aligned}
\sup _{x \in \mathbb{R}^{n}, r>0} & \frac{1}{\varphi_{2}(x, r) \Phi^{-1}\left(r^{-n}\right)} \\
& \times \int_{r}^{\infty} \underset{t<s<\infty}{\operatorname{essinf}} \varphi_{1}(x, s) \Phi^{-1}\left(t^{-n}\right) \frac{d t}{t}<\infty,
\end{aligned}
$$

where $C$ does not depend on $x$ and $r$. Then, a $\Phi$-admissible sublinear singular operator $T$ is bounded from $M_{\Phi, \varphi_{1}}\left(\mathbb{R}^{n}\right)$ to $M_{\Phi, \varphi_{2}}\left(\mathbb{R}^{n}\right)$ and a weak $\Phi$-admissible sublinear singular operator $T$ is bounded from $M_{\Phi, \varphi_{1}}\left(\mathbb{R}^{n}\right)$ to $W M_{\Phi, \varphi_{2}}\left(\mathbb{R}^{n}\right)$. 
Proof. By Lemma 15 and Theorem 13 with $v_{1}(r)=\varphi_{1}(x, r)^{-1}$, $v_{2}(r)=1 / \varphi_{2}(x, r) \Phi^{-1}\left(r^{-n}\right), g(r)=\|f\|_{L_{\Phi}(B(x, r))}, \omega(r)=$ $\Phi^{-1}\left(r^{-n}\right) / r$, we have

$$
\begin{aligned}
\|T f\|_{M_{\Phi, \varphi_{2}}} & =\sup _{x \in \mathbb{R}^{n}, r>0} \varphi_{2}(x, r)^{-1}\|T f\|_{L_{\Phi}(B(x, r))} \\
\lesssim & \sup _{x \in \mathbb{R}^{n}, r>0} \frac{1}{\varphi_{2}(x, r) \Phi^{-1}\left(r^{-n}\right)} \\
& \times \int_{r}^{\infty}\|f\|_{L_{\Phi}(B(x, t))} \Phi^{-1}\left(t^{-n}\right) \frac{d t}{t} \\
\lesssim & \sup _{x \in \mathbb{R}^{n}, r>0} \varphi_{1}(x, r)^{-1}\|f\|_{L_{\Phi}(B(x, r))}=\|f\|_{M_{\Phi, \varphi_{1}}}, \\
= & \sup _{x \in \mathbb{R}^{n}, r>0} \varphi_{2}(x, r)^{-1}\|T f\|_{W L_{\Phi}(B(x, r))} \\
\lesssim & \sup _{x \in \mathbb{R}^{n}, r>0} \frac{1}{\varphi_{2}(x, r) \Phi^{-1}\left(r^{-n}\right)} \\
& \times \int_{r}^{\infty}\|f\|_{L_{\Phi}(B(x, t))} \Phi^{-1}\left(t^{-n}\right) \frac{d t}{t} \\
\vdots & \sup _{x \in \mathbb{R}^{n}, r>0} \varphi_{1}(x, r)^{-1}\|f\|_{L_{\Phi}(B(x, r))}=\|f\|_{M_{\Phi, \varphi_{1}}}
\end{aligned}
$$

Note that, from Theorems 8, 9, and 18 we get the following corollaries, which are proven in [15].

Corollary 19. Let $\Phi$ be any Young function and $\varphi_{1}, \varphi_{2}$, and $\Phi$ satisfy condition (49). Then the maximal operator $M$ is bounded from $M_{\Phi, \varphi_{1}}\left(\mathbb{R}^{n}\right)$ to $W M_{\Phi, \varphi_{2}}\left(\mathbb{R}^{n}\right)$ and for $\Phi \in \nabla_{2}$, the operator $M$ is bounded from $M_{\Phi, \varphi_{1}}\left(\mathbb{R}^{n}\right)$ to $M_{\Phi, \varphi_{2}}\left(\mathbb{R}^{n}\right)$.

Corollary 20. Let $\Phi$ be any Young function and $\varphi_{1}, \varphi_{2}$, and $\Phi$ satisfy the condition (49). Then, the singular integral operator $T$ is bounded from $M_{\Phi, \varphi_{1}}\left(\mathbb{R}^{n}\right)$ to $M_{\Phi, \varphi_{2}}\left(\mathbb{R}^{n}\right)$ for $\Phi \in \Delta_{2} \cap \nabla_{2}$ and from $M_{\Phi, \varphi_{1}}\left(\mathbb{R}^{n}\right)$ to $W M_{\Phi, \varphi_{2}}\left(\mathbb{R}^{n}\right)$ for $\Phi \in \Delta_{2}$.

\section{Conflict of Interests}

The author declares that there is no conflict of interests regarding the publication of this paper.

\section{Acknowledgments}

The author would like to express their gratitude to the referee for his very valuable comments and suggestions. Thanks to referee we added the Remark 2.

\section{References}

[1] C. B. Morrey, Jr., "On the solutions of quasi-linear elliptic partial differential equations," Transactions of the American Mathematical Society, vol. 43, no. 1, pp. 126-166, 1938.
[2] E. M. Stein, Harmonic Analysis: Real Variable Methods, Orthogonality and Oscillatory Integrals, vol. 43, Princeton University Press, Princeton, NJ, USA, 1993.

[3] A. Torchinsky, Real-Variable Methods in Harmonic Analysis, vol. 123 of Pure and Applied Mathematics, Academic Press, New York, NY, USA, 1986.

[4] W. Orlicz, "Über eine gewisse Klasse von vom typus B," Bulletin de L’Académie Polonaise Des Sciences, pp. 207-220, 1932, reprinted in: Collected Papers, PWN, Warszawa, pp. 217-230, 1988.

[5] W. Orlicz, “Über Räumen $\left(L^{M}\right)$," Bulletin de L'Académie Polonaise Des Sciences, pp. 93-107, 1936, reprinted in: Collected Papers, PWN, Warszawa, pp. 345-359, 1988.

[6] M. A. Krasnoselskii and J. B. Rutickii, Convex Functions and Orlicz Spaces, P. Noordhoff, Groningen, The Netherlands, 1961.

[7] "Harmonic analysis," in Proceedings of the ICM 90 Satellite Conference, S. Igari, Ed., pp. 183-189, Springer, Tokyo, 1991.

[8] R. O'Neil, "Fractional integration in Orlicz spaces. I," Transactions of the American Mathematical Society, vol. 115, pp. 300328, 1965.

[9] H.-o. Kita, "On maximal functions in Orlicz spaces," Proceedings of the American Mathematical Society, vol. 124, no. 10, pp. 3019-3025, 1996.

[10] H. Kita, "On Hardy-Littlewood maximal functions in Orlicz spaces," Mathematische Nachrichten, vol. 183, pp. 135-155, 1997.

[11] A. Cianchi, "Strong and weak type inequalities for some classical operators in Orlicz spaces," Journal of the London Mathematical Society, vol. 60, no. 1, pp. 187-202, 1999.

[12] E. Nakai, "Generalized fractional integrals on Orlicz-Morrey spaces," in Banach and Function Spaces, pp. 323-333, Yokohama, Yokohama, Japan, 2004.

[13] E. Nakai, "Calderón-Zygmund operators on Orlicz-Morrey spaces and modular inequalities," in Banach and Function Spaces II, pp. 393-410, Yokohama, Yokohama, Japan, 2008.

[14] E. Nakai, "Orlicz-Morrey spaces and the Hardy-Littlewood maximal function," Studia Mathematica, vol. 188, no. 3, pp. 193221, 2008.

[15] F. Deringoz, V. S. Guliyev, and S. Samko, "Boundedness of maximal and singular operators on generalized Orlicz-Morrey spaces," Advances in Harmonic Analysis and Operator Theory, vol. 235, pp. 1-24, 2013.

[16] Y. Sawano, S. Sugano, and H. Tanaka, "Orlicz-Morrey spaces and fractional operators," Potential Analysis, vol. 36, no. 4, pp. 517-556, 2012.

[17] R. Vodák, "The problem $\nabla \cdot v=f$ and singular integrals on Orlicz spaces," Acta Universitatis Palackianae Olomucensis, vol. 41, pp. 161-173, 2002.

[18] M. M. Rao and Z. D. Ren, Theory of Orlicz Spaces, vol. 146, Marcel Dekker, New York, NY, USA, 1991.

[19] H. Kita, "Inequalities with weights for maximal functions in Orlicz spaces," Acta Mathematica Hungarica, vol. 72, no. 4, pp. 291-305, 1996.

[20] V. Kokilashvili and M. Krbec, Weighted Inequalities in Lorentz and Orlicz Spaces, World Scientific, Singapore, 1991.

[21] I. Genebashvili, A. Gogatishvili, V. Kokilashvili, and M. Krbec, Weight Theory for Integral Transforms on Spaces of Homogeneous Type, vol. 92, Longman, Harlow, UK, 1998.

[22] V. S. Guliyev, Integral operators on function spaces on the homogeneous groups and on domains in $\mathbb{R}^{n}$ [Ph.D. thesis], Steklov Mathematical Institute, Moscow, Russia, 1994, (Russian). 
[23] V. S. Guliyev, Function Spaces, Integral Operators and Two Weighted Inequalities on Homogeneous Groups, Some Applications, Baku, Azerbaijan, 1999, (Russian).

[24] V. S. Guliyev, "Boundedness of the maximal, potential and singular operators in the generalized Morrey spaces," Journal of Inequalities and Applications, vol. 2009, Article ID 503948, 20 pages, 2009.

[25] V. S. Guliyev, S. S. Aliyev, T. Karaman, and P. S. Shukurov, "Boundedness of sublinear operators and commutators on generalized Morrey spaces," Integral Equations and Operator Theory, vol. 71, no. 3, pp. 327-355, 2011.

[26] A. Akbulut, V. Guliyev, and R. Mustafayev, "On the boundedness of the maximal operator and singular integral operators in generalized Morrey spaces," Mathematica Bohemica, vol. 137, no. 1, pp. 27-43, 2012.

[27] V. S. Guliyev, "Local generalized Morrey spaces and singular integrals with rough kernel," Azerbaijan Journal of Mathematics, vol. 3, no. 2, pp. 79-94, 2013.

[28] V. S. Guliyev, "Generalized local Morrey spaces and fractional integral operators with rough kernel," Journal of Mathematical Sciences, vol. 193, no. 2, pp. 211-227, 2013. 


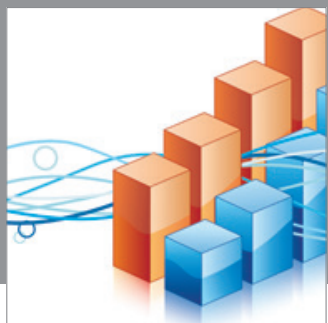

Advances in

Operations Research

mansans

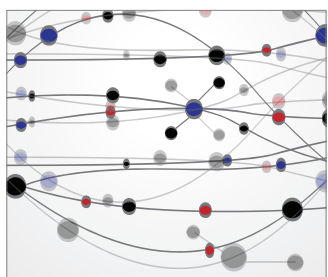

The Scientific World Journal
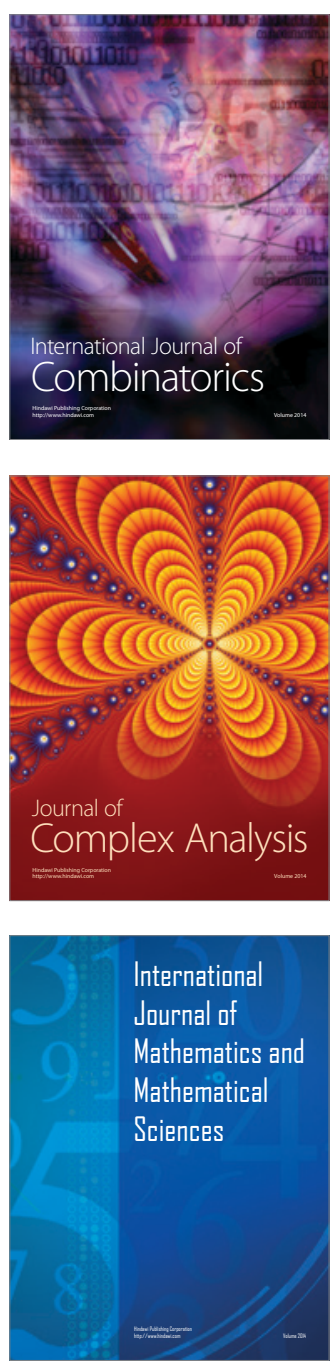
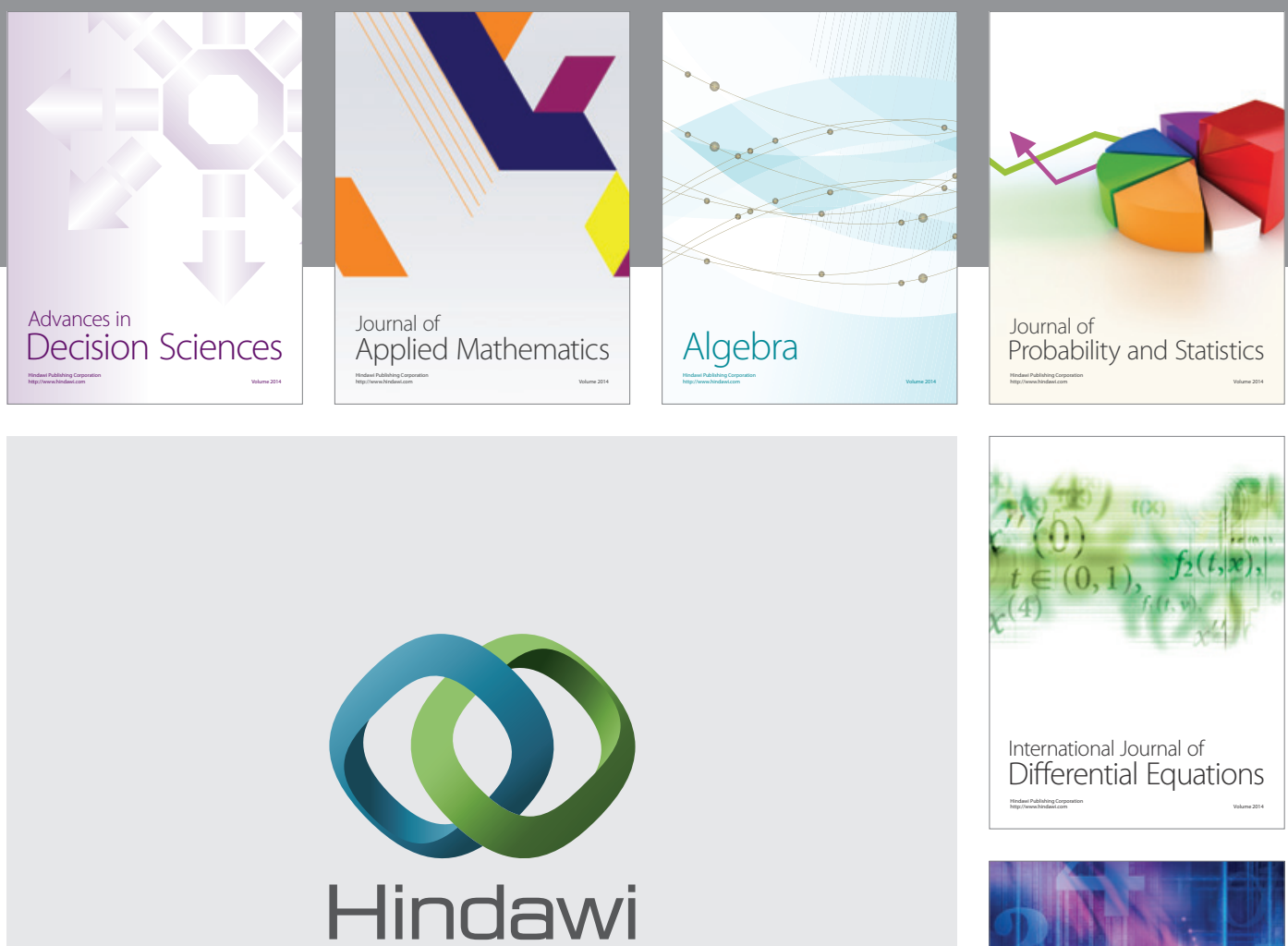

Submit your manuscripts at http://www.hindawi.com
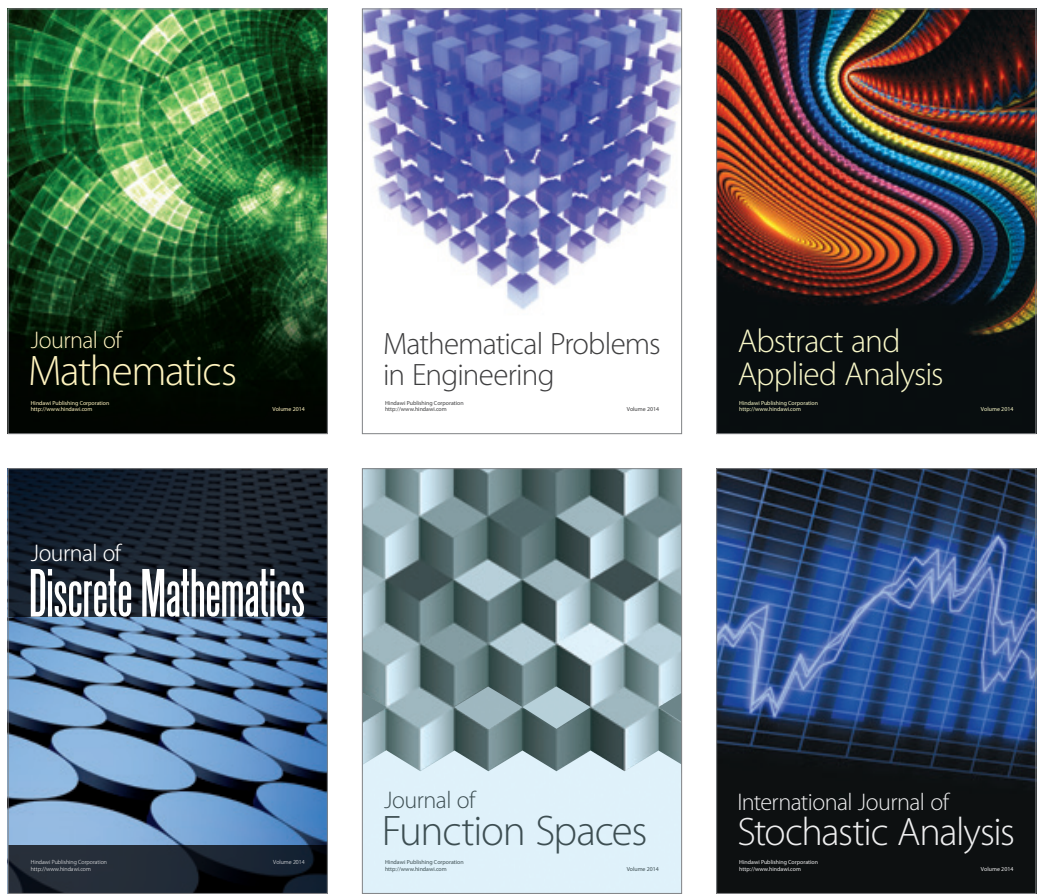

Journal of

Function Spaces

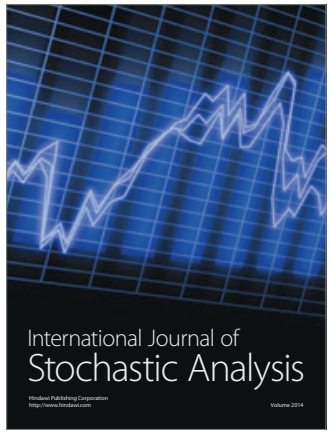

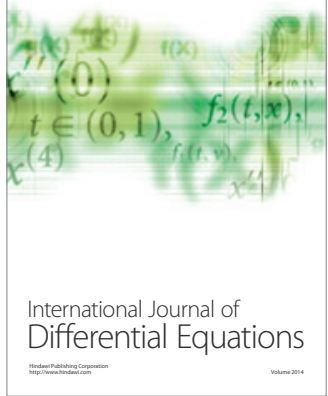
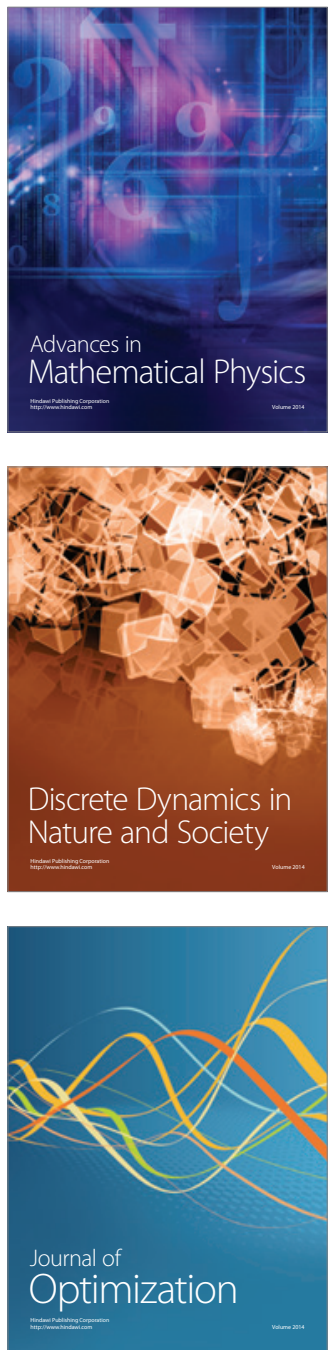